\title{
Escaping from ESKAPE. Clinical Significance and Antibiotic Resistance Mechanisms in Acinetobacter baumannii: a Review
}

\author{
Corneliu Ovidiu Vrancianu ${ }^{1,2} \mathbb{D}$, Cristina Florentina Pelcaru ${ }^{1,2}$, Alexandru Alistar ${ }^{1}$, Irina \\ Gheorghe $^{1,2^{*} \mathbb{D}}$, Luminita Marutescu ${ }^{1,2} \mathbb{( \mathbb { D }}$, Marcela Popa ${ }^{2 \mathbb{D}}$, Ilda Czobor ${ }^{1,2} \mathbb{D}$,

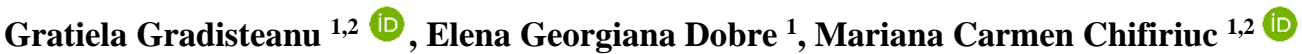

1 University of Bucharest, Faculty of Biology, Microbiology-Immunology Department, Bucharest, Romania; ovidiu.vrancianu@yahoo.com (C.O.V.); cristina.pelcaru@yahoo.com (C.F.P); alexandru_alistar@yahoo.com (A.A.); lumi.marutescu@gmail.com (L.M.); bmarcelica@yahoo.com (M.P.); $\quad$ ilda.czobor@yahoo.com (I.C.); gratiela87@gmail.com (G.G.); dobregeorgiana_95@yahoo.com (E.G.D.); carmen.chifiriuc@ bio.unibuc.ro (M.C.C.)

2 The Research Institute of the University of Bucharest, Romania

* Correspondence: iryna_84@yahoo.com (I.G.);

Scopus Author ID 55036488700

Received: 2.07.2020; Revised: 18.07.2020; Accepted: 19.07.2020; Published: 22.07.2020

\begin{abstract}
Antibiotic resistance represents a critical threat in clinical settings nowadays, with an essential ecological dimension. Due to the involvement of the resistance genes, this phenomenon has gained an unprecedented expansion. Their accumulation and dissemination are facilitated by mobile genetic elements (MGEs) (plasmids, transposons, integrons, genomic islands) that can increase intracellular DNA mobility. In clinical settings, one of the critical resistant bacteria associated with nosocomial infections is Acinetobacter baumannii. This Gram-negative bacterium exhibits variate resistance mechanisms that enable it to survive in extreme environmental conditions and to evade antimicrobial agents. The enormous adaptive capacity and the essential role in the emergence of severe nosocomial infections lead to the need to study more deeply the mechanisms involved in antibiotic resistance in A. baumannii strains. In this review, we will initially present the role of A. baumannii in human and veterinary infectious pathology. We will subsequently discuss the main genetic resistance mechanisms (both intrinsic and acquired) encountered in A. baumannii strains.
\end{abstract}

Keywords: resistance; Acinetobacter baumannii; nosocomial infections; $\beta$-lactamase; antibiotic.

(C) 2020 by the authors. This article is an open-access article distributed under the terms and conditions of the Creative Commons Attribution (CC BY) license (https://creativecommons.org/licenses/by/4.0/).

\section{Introduction}

According to the World Health Organization, one of the critical global warning threatening human health is represented by antimicrobial resistance (AR) [1]. The most common and severe MDR pathogens have been called within the acronym "ESKAPE" Enterococcus faecium, Staphylococcus aureus, Klebsiella pneumoniae, Acinetobacter baumannii, Pseudomonas aeruginosa, and Enterobacter spp. [2]. Among opportunistic bacteria, Acinetobacter baumannii is a critical concern both for nosocomial and communityacquired infections, mainly due to its diverse antibiotic resistance mechanisms [3]. A. baumannii is part of the A. baumannii - A. calcoaceticus complex (Acb), initially including four species: A. calcoaceticus, A. baumannii, A. nosocomialis, and A. pittii [4]. Subsequently, several other species have been proposed for inclusion in this complex: A. seifertii [5], A. lactucae [6], and Acinetobacter species "between 1 and 3" [7]. The prolonged resistance to 
carbapenems and other antibiotics makes the Acb complex one of the biggest challenges in hospitals, drastically decreasing the effectiveness of the current treatment options. Acinetobacter spp. have been implicated in several pathologies such as pneumonia, urinary tract infections, skin and wound infections, infective endocarditis, bacteremia, and secondary meningitis [7]. The colonization and infection with multidrug-resistant (MDR) $A$. baumannii are influenced by admission to ICUs, mechanical ventilation, exposure to antimicrobial agents, and extended periods of hospitalization [2,8]. The severity of the infections is caused by the high ability of this bacterium to survive in extreme environmental conditions through the multitude of resistance mechanisms. A. baumannii revealed multiple resistance mechanisms, which are based on mutations and the acquisition of ARGs through horizontal gene transfer (HGT) (MUNITA \& ARIAS [9]. In this review, we will present a short general characterization of Acinetobacter species, and the role of A. baumannii in human and veterinary infectious pathology. Subsequently, we will discuss the main genetic resistance mechanisms (bot intrinsic and acquired) encountered in A. baumannii strains.

\section{A. baumannii - general features}

Acinetobacter was frequently ignored in the 1960s when isolated from clinical samples, being considered a commensal, opportunist, relatively low-grade pathogen. For the past 30 years, interest in this species has been growing, one of the main reasons being the emergence of MDR strains, involving most first-line antibiotic classes. Nowadays, there is a multitude of studies on 'infections and resistant Acinetobacter' in the international scientific literature.

Identification of Acinetobacter through phenotypic traits and chemotaxonomic methods, to the individual species level, is difficult due to clusters of closely related species. There have been described at least 40 genospecies of Acinetobacter [10] identified through DNA-DNA hybridization, including the genospecies 1 - A. calcoaceticus, 2- A. baumannii, 4 - A. haemolyticus, 5 - A. junii, 7 - A. johnsonii, 8 - A. lwoffii and 12 - A. radioresistens. Most other genospecies cannot be separated easily with conventional biochemical tests or commercial identification systems [11]. Among these species, A. calcoaceticus, A. baumannii, Acinetobacter genomic species 3, and Acinetobacter genomic species $13 \mathrm{TU}$ are very closely related and are difficult to separate from each other phenotypically. Consequently, they have been distributed in A. calcoaceticus - baumannii complex [4]. This nomenclature can be misleading because A. calcoaceticus has not been involved in clinical diseases, in contrast to the other three species that are clinically significant being involved, especially in nosocomial infections [2]. 80\% of the clinical infections caused by Acinetobacter spp. are associated with this group [4]. The deficiency of a robust phylogenetic scenario encompassing all known Acinetobacter spp. as well as the unknown position of their last common ancestor severely restrains the understanding of the diversification of this genus [5].

Solid media often used in clinical microbiology laboratories (sheep blood agar or tryptic soy agar) are used to recover Acinetobacter spp. of human origin. Colonies of the $A$. calcoaceticus - baumannii complex, are 1.5 to $3 \mathrm{~mm}$ diameter, in contrast with many other Acinetobacter species producing smaller and more clear colonies. A. haemolyticus and other species not well-defined show hemolysis on sheep blood agar media. This characteristic is not present in Acinetobacter isolates belonging to the A. calcoaceticus - baumannii complex [3]. 


\section{Habitat}

Species belonging to the genus Acinetobacter spp. are ubiquitous; they being recovered from almost all environmental samples. However, some different species of the genus are generally associated with different habitats. Acinetobacter genomospecies 3 is founded in water, soil, vegetable products, and colonizes the human skin. A. johnsonii and A. haemolyticus are found in water, wastewater, soil, in human feces, and human skin. A. lwoffii and $A$. radioresistens are colonizers of the human skin. Acinetobacter genomic species 11 is found in water, soil samples, and in human microbiota [12].

It can be dominant in the microbial communities found in numerous habitats, including the plant phyllosphere and rhizosphere, bioaerosols, oil, sand, and other hydrocarboncontaining environments [13]. In contrast to other bacteria causing healthcare-associated infections (HAIs), this genus species can survive on dry surfaces long periods.

According to the last researches, not all species of the genus Acinetobacter are found naturally in the environment. Some of them, as A. schindleri and A. ursingii, have been recovered only from human specimens. Some Acinetobacter spp. were identified as part of the commensal microbiota, being recovered from dry and moist areas of healthy human skin, such as the axilla, groin, and toe webs, the oral cavity and the respiratory tract [11]. According to previous data, the human skin and mucous membranes colonization by Acinetobacter spp. strains were reported by up to $44 \%$ of out-patients and up to $75 \%$ of in-patients [14].

Other species such as A. baylyi, A. grimontii, A. tjernbergiae, A. bouvetii, A. towneri, and $A$. tandoii, isolated from activated sludge, have not been found in human samples. Acinetobacter spp. have been associated with food contamination, being recovered from vegetables, apples, melons, cabbages, cauliflowers, lettuce, cucumbers, peppers, mushrooms, radishes, carrots as well as tubers such as potatoes and cereals such as sweet corn [12].

A. baumannii, A. haemolyticus, A. lwoffii, and A. calcoaceticus cam form thin layers of microorganisms on glass, medical tools, metals, ceramics, and other inanimate objects, through Pilus-mediated biofilms (PMB) [12]. In the hospital settings, there have been isolated from reusable medical equipment such a ventilator, tubings, arterial pressure monitoring devices, humidifiers, washbasins, plastic urinals, and respirometers.

\section{Adaptative mechanisms of A. baumannii to different environments}

A. baumanii is not a ubiquitous organism. Despite its association with skin infections, A. baumannii is rarely found as part of the healthy skin microbiota. In one study estimating that the bacterium colonizes only $3 \%$ of the population. A key risk group for infection with $A$. baumanii is represented by the armed forces deployed to conflict zones, such as Iraq, earning A. baumannii, the notorious name of 'Iraqibacter' [15]. The extreme environmental conditions related with these desert campaigns offered a propitious environment for the A. baumannii, becoming the main cause of infection among injured soldiers. The study conducted by the US Navy hospital ship USNS Comfort (T-AH-20), revealed that $4.1 \%$ of all skin and soft-tissue infections encountered were related to A. baumannii (axilla, groin and toe webs being the areas of highest colonization) [2].

The ability to survive in hospital environments lead A. baumannii to become one of the most successful nosocomial pathogens [16]. Surviving as a commensal on the skin or hair of hospital staff and patients and colonizing a variety of body surfaces, A. baumannii is considered widespread in the clinical environments. Various contaminated objects have been identified 
and could serve as potential reservoirs for this nosocomial pathogen. A. baumannii can adhere and colonize indwelling devices such as catheters and respiratory equipment, mattresses, pillows as well as biotic surfaces such as those of human epithelial cells [17].mattresses, pillows Human utility articles, like computers, mouse, and gloves, and pets, have been suggested as causes for the spread of $A$. baumannii strains. The identification of reservoir sources of A. baumannii strains is significant for the control measures [16].

A. baumannii can survive exposure to common disinfectants - chlorhexidine, phenols, gluconate, on fingertips or dry surfaces, and to long nutrient, starvation due to its ability to develop biofilms [18].

\subsection{Human and veterinary infectious pathology}

Once considered, organisms of low clinical significance Acinetobacter spp. (mainly A. baumannii, being followed by A. haemolyticus and A. lwoffii) have nowadays emerged as important causes of nosocomial infections due to the selection of MDR strains, following the introduction of new antibiotics in the clinical practice and agriculture and the use of invasive procedures in ICUs [12]. Acinetobacter spp. have been involved in several pathologies such as ventilator-associated pneumonia, urinary tract infections, skin and wound infections, infective endocarditis, bacteremia, and secondary meningitis [7]. Incidence and mortality due to $A$. baumannii strains are higher than those due to A. pittii or A. nosocomialis; however, the last two are frequently isolated from nosocomial infections. These bacteria have been compared to methicillin-resistant Staphylococcus aureus (MRSA), being named the 'Gram-negative MRSA'. The incidence of Acinetobacter causing bloodstream infection has been estimated to be about ten-fold less than that of S. aureus (1.5\% vs. 14\%) [19].

\subsubsection{Nosocomial infections}

A. baumannii is a critical pathogen being responsible for hospital-acquired nosocomial infections. However, Acinetobacter spp. is considered to be a low-grade pathogen, and can remain on or in the human body without causing illness. When infections become apparent, the number of colonized patients is already high; in this case, taking precautions to prevent an outbreak is too late. All surfaces in the environment can represent reservoirs for Acinetobacter, once an outbreak is established. These organisms are robust survivors, especially in ICU settings. They can survive for weeks in dry conditions [19].

There is a significant risk if A. baumannii is identified in clinical settings, especially in ICUs, where patients are chronically ill, being therefore immunocompromised and spending a prolonged period in the hospital unit. Also, at risk of developing A. baumannii infections are the patients undergoing surgery, with endotracheal tubes and intravascular, ventricular, or urinary catheters, sutures, ventilators, and those under dialysis or antimicrobial therapy within the past 90 days. Another risk factor for the acquisition of Acinetobacter is represented by the presence and duration of invasive procedures. Another pathway that can lead to the development of nosocomial pneumonia or bacteremia involves bacterial overgrowth in the stomach. This process may occur under conditions of reduced acid secretion in the stomach, such as occurs in many ICU patients [19].

Sites for colonization are represented by the respiratory tract, blood, pleural fluid, urinary tract, surgical wounds, central nervous system, skin, and eyes. The two most common clinical manifestations of $A$. baumannii strains are nosocomial pneumonia and bacteremia. The 
A. baumannii strains with a biofilm-forming ability that can produce biofilms on the surface of the endotracheal tube may pose a serious concern to those patients who require mechanical ventilation. Consequently, this can lead to the relatively high levels of colonization in the lower part of the respiratory tract (Fig. 1) [2].

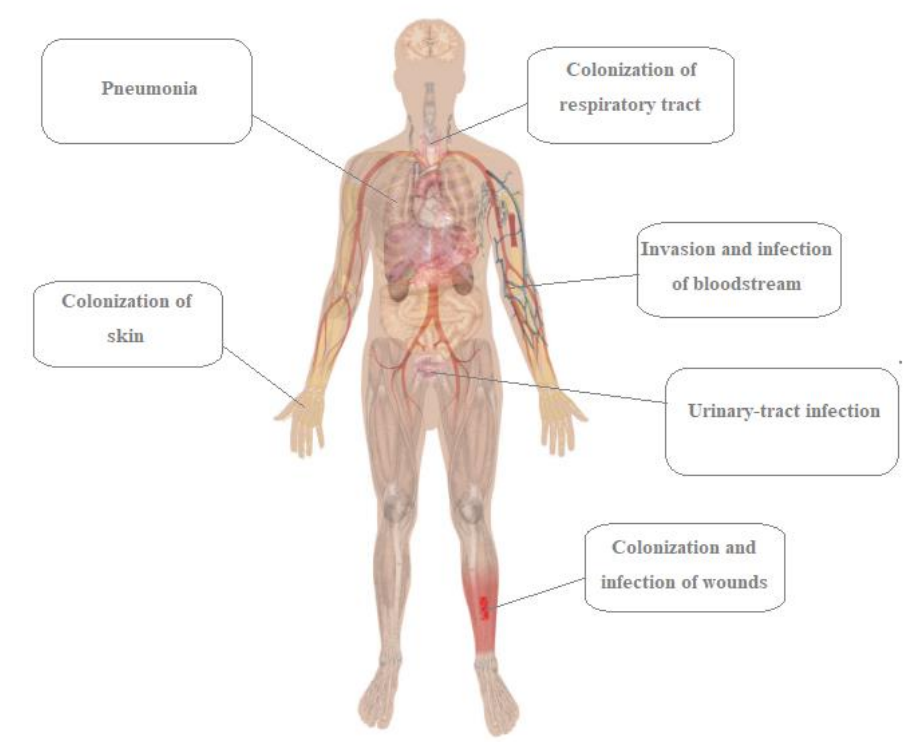

Figure 1. Colonization sites and infections produced by A. baumannii.

The skin and moist mucous membranes exposed through accident or injury and infected by A. baumannii present a "peau d'orange" appearance (skin orange-like) followed by a sandpaper-like appearance, with translucent vesicles on the skin. In skin disruption zones, it may be seen hemorrhagic bullae and a visible necrotizing process associated with bacteremia. Failure to treat this infection can lead to septicemia and death. Although A. baumannii is likely responsible for these recognizable features, co-pathogens like Klebsiella pneumoniae, Candida albicans, and Enterococcus faecalis, are considered a contributing factor. These co-pathogens may be responsible for necrotizing infection and may create a gateway into the bloodstream for A. baumannii strains [2].

A characteristic manifestation of nosocomial A. baumannii is the wound infection, that could be associated with natural or human-made disasters, such as the 1999 Marmara earthquake, the 2002 Bali bombing, and military operations. A strikingly high number of deepwound infections, burn wound infections, and osteomyelitis cases were associated with victims of the Iraq conflict. Isolates often exhibited multidrug resistance [20]. Nosocomial infections caused by other species, such as A. johnsonii, A. junii, A. lwoffii, A. parvus, A. radioresistens, A. schindleri, and $A$. ursingii, are rare and are limited to catheter-related bloodstream infections. Mortality is very low, and their clinical course is usually benign.

\subsubsection{Community-acquired infections}

A. baumannii is not considered a community pathogen, but in immunocompromised individuals and children, it populates tracheostomy sites and can cause community-acquired bronchiolitis and tracheobronchitis [21].

The most frequent community-acquired infection involving Acinetobacter spp. is acute pneumonia, predominantly occurring in warm, humid, tropical environments, especially in Australia, Oceania, and Asia, including China, Taiwan, and Thailand [22]. 
Since the 1980s, about 100 cases of community-acquired pneumonia cases caused by Acinetobacter spp. have been described. Meningitis, cellulitis, or primary bacteremia has also been reported but more rarely. Patients with acute pneumonia generally have a history of alcohol abuse, diabetes, cancer, or bronchopulmonary disease. The course of infection can be fulminant, with septic shock in nearly $30 \%$ of cases, as well as respiratory failure. Patients generally have productive sputum with hemoptysis. The high mortality rate has been related to the background of the patient and delays in initiating appropriate therapy $[19,23]$.

\subsubsection{Veterinary infections}

A. beijerinkii and A. baumannii are among the species of Acinetobacter genera involved in animal diseases. Different studies reported chicken, turkeys, and calves septicemia, mastitis and metritis in cows, abortions in cattle, pigs and horses, keratoconjunctivitis in cattle, omphalitis in calves, ear infections in cats and respiratory infections and Balanoposthitis in horses [12].

Infections due to A. baumannii have rarely been reported in animals. In 2000, Vaneechoutte et al. [24] reported the isolation of A. baumannii strains from jugular catheter tips collected from horses suffering from a variety of conditions and hospitalized in the Ghent University equine clinic. Four of the horses were hospitalized for colic surgery, and three were hospitalized for treatment of enteritis.

Francey et al., in 2000 [25] described 19 cases of A. baumannii infection from an ICU

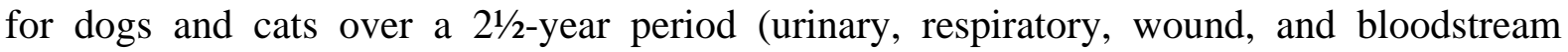
infections). They demonstrated that $A$. baumannii can cause life-threatening hospital-acquired infections in small animals and can limit the success of intensive care as well as routine procedures. With a mortality rate of $100 \%$ in systemically infected patients, it also influenced treatment outcomes.

First molecular analysis of $A$. baumannii strains responsible for infections in pets and horses using established methods previously used for human isolates was made by Endimiani et al. in 2011 [26]. They concluded that shortly, veterinary A. baumannii isolates could acquire further mechanisms of resistance and evolve into pathogens more challenging to treat. Thereby, more extensive screening and epidemiological studies should be performed to investigate the impact of animals on the spread of MDR A. baumannii isolates among humans.

\section{Antibiotic resistance mechanisms in Acinetobacter baumannii}

The severity of A. baumannii infections is caused by the high ability of this bacterium to survive in extreme environmental conditions through the multitude of resistance mechanisms $[27,28]$. A . baumannii revealed both intrinsic and acquired resistance mechanisms, which are based on chromosomal mutations and the acquisition of ARGs through HGT [9]. AR of $A$. baumannii is driven by multiple mechanisms: drug inactivation/alteration, modification of drug binding sites/targets, cell permeability modification, and biofilm formation [29].

\subsection{Intrinsic resistance (IR)}

There are several antibiotic classes towards which A. baumannii posses IR, such as $\beta$ lactams, macrolides, trimethoprim, or fosfomycin [30]. Mechanisms of IR include the existence of natural membrane impermeability, the basal activity of efflux pumps, and the presence of chromosomally encoded OXA-51 oxacylinase, and of ADC cephalosporinase [31-33]. The 
efflux pumps contribute to the $\beta$-lactams, tetracyclines, fusidic acid, and tigecycline resistance [34]. Outer membrane permeability is an important mechanism involved in AR and virulence of A. baumannii strains. OmpA porin is associated with aztreonam, chloramphenicol, and nalidixic acid resistance [35]. More recent studies have also highlighted the association of OmpA with lung infections, sepsis, and high mortality [36,37].

\subsection{Biofilm and resistance}

Biofilms are sessile microbial cells embedded in a self-producing exopolysaccharides (EPS) matrix. In biofilms, bacterial cells possess increased resistance to antibiotics or biocides, the host's immune response, antimicrobial agents, and a high ability to survive in extreme environmental conditions [38]. The impaired diffusion of antimicrobial agents causes increased bacterial resistance in biofilms due to microbial aggregation, overexpression of matrix exopolymers, and alteration of phenotypic and genotypic microbial characteristics to stress response $[39,40]$. The phenotypic and genotypic characteristics of bacterial cells are triggered when producing quorum sensing (QS) signals in a cell-dependent manner, signals that allow communication between cells when significant changes in environmental conditions occur [41]. Antimicrobial agents such as antibiotics or biocides can trigger the formation of biofilms if administered at concentrations lower than the minimum inhibitory concentration (MIC) [42]. Therefore, treating infections caused by biofilm-forming bacteria requires higher doses of antibiotics and antimicrobial agents [43]. Also, an association was observed between the increased resistance to biocides and the ability to form biofilms, in parallel with the increased activity of efflux pumps [44-46]. Both Gram-negative and Gram-positive bacterial biofilms are highly resistant, requiring high concentrations of biocides for treatment, suggesting that biofilm formation is a natural mechanism of resistance to the action of antimicrobial agents.

The interaction between biofilms and antimicrobial agents is not fully understood. This is especially so in terms of cross-resistance phenomena, which represent an additional challenge to prevent the formation of biofilms and eradicate existing ones.

\subsection{Acquired resistance to horizontal gene transfer}

The primary mechanism of acquired resistance is represented by mobile genetic elements (MGE). MGEs are fragments of DNA that encode enzymes and other proteins involved in intracellular or intercellular mobility. Intracellular mobility occurs within the same cell, from a chromosome to a plasmid or between plasmids. Transposons (Tn), except the conjugative ones, insertion sequences (IS), and integrons, belong to the intracellular MGE [47]. Intercellular mobility occurs through MGEs able of self-replication and conjugative transfer, such as plasmids and conjugative Tn. MGE achieve mobilization through transformation [48-51].

Plasmids play an essential role in the accumulation and transfer of ARGs. Especially in the case of Gram-negative bacteria, plasmids mediate the development and spread of AR to most known antibiotics, including $\beta$-lactams, aminoglycosides (AMGs), tetracyclines, chloramphenicol, sulfonamides, trimethoprim, macrolides, polymyxins and quinolones $[52,53]$. Usually, the plasmids involved in the resistance phenomenon are conjugative, a property that gives them the ability to support both their transfer and the transfer of other plasmids. They have mechanisms to control the number of copies and the replication process [51]. In A. baumannii, PCR-based on replicon typing methodology, there have been described 
27 replicase genes classified into 19 homology groups on the basis of their nucleotide homology [54].

The insertion sequences (IS) are simple transposable elements with dimensions between 0.7 and $2.5 \mathrm{~kb}$. The presence of two copies of the same IS on either side of an ARG forms a complex structure called a compound transposon, which is essential in the dissemination of ARGs [55]. The role of IS in AR is due both to their ability to transfer ARGs and to mediate the expression of these genes by integrating them into their structure, or by providing an active promoter $[49,56]$. In A. baumannii, there are several IS involved in increasing the level of AR. ISAbal, ISAba2, ISAba3, ISAba4, and IS 18 are commonly associated with the expression of carbapenemase encoding genes [57]. Poirel et al. [58] observed that ISAbal is involved in the dissemination of blaOXA-23 gene from A. radioresistens to A. baumannii. Although ISAbal is considered exclusively in A. baumannii, Segal et al. reported the presence of ISAbal in A. lwoffii, which demonstrates that these elements have high mobility [59]. ISAbal has also been identified upstream of the bla OxA-23-like, bla OXA-51-like, blaOXA-58-like, and bla $a_{\mathrm{AmpC}}$ genes, where it acts as a promoter sequence and increases the expression of ARGs [60]. In the case of the blaoxA-23 and blaoxA-51 genes, the presence of ISAbal upstream of these genes is necessary to encode carbapenem resistance [61]. More recently, there have been reported ISAbalO upstream of the blaoxA-23 gene. The presence of ISAba10 upstream blaoXA-23 gene has been reported to increase its expression by up to 5-fold, by providing an additional promoter [62]. In A. baumannii, IS has also been associated with metallo- $\beta$-lactamases (MBL) from NDM type. It was observed that the bla NDM gene is placed between two copies of the ISAba125 in the structure of a compound Tn called Tn125 [58]. Similar to other IS, ISAba125 provides a promoter, increasing the expression of the bla NDM gene. Robledo et al. reported the role of ISEcpl in the mobilization of ARGs such as blactX-M, qnrB19, rmtC, bla $a_{\mathrm{ACC}-1}$ and bla ${ }_{\mathrm{CMY}}$ [63]. In conclusion, IS are genetic elements with an essential role in increasing the expression and dissemination of ARGs in A. baumannii.

Transposons are part of the MGEs category that can mobilize ARGs both intramolecularly and intermolecularly. There are two categories of transposons: compounds, which have a central gene flanked by two IS elements; complex Tn, which contain the tnpA gene encoding transposase, the tnpR gene encoding resolvase, and one or more cargo genes [64]. In A. baumannii, transposons contain genes essential for AR, such as blaoxA-23. Studies have shown that three main transposons are involved in the dissemination of the blaoxA-23 gene: Tn2006, Tn2007, and Tn2008. In Tn2006, the blaoxA-23 gene is flanked by two copies of ISAba1, and in the case of Tn2008, there is only one copy. In Tn2007, the blaoxA-23 gene is associated with a copy of ISAba4, located upstream of the gene [65]. In terms of carbapenem resistance, Tn2006 is considered the most common mechanism [62].

Integrons are natural cloning and expression systems that can embed open-reading frames (ORFs) by site-specific recombination and transform them into functional genes in the presence of a promoter [66]. Integrons play an essential role in the acquisition and dissemination of ARGs, both in the case of Gram-negative [67] and Gram-positive bacteria (CAMERANESI et al. [68]). There are five classes of integrons, of which the first three are associated with MDR phenotypes, having an essential role in the dissemination of ARGs [69]. In $A$. baumannii, class 1 integrons are more represented, having high prevalence in the isolates reported from Europe, Asia, and America [70]. This class can be associated with transposons derived from Tn402 and can be inserted into large transposons such as Tn21. Also, class 1 integrons are associated with several IS such as IS26, IS1999, IS2000, and IS6100 [71]. Studies 
have also shown the association of this class of integrons with different classes of antibiotics. Mendes et al. highlighted the association of class 1 integrons with MBL, such as bla IMP-1. All the analyzed isolates presented this class of integrons called In86. In86 presents in its structure a gene box composed of bla IMP-1, aac( $\left.6^{\prime}\right)-3 \mathrm{I}$, and aadA1. The aac gene (6 ')-31 encodes the most AMGs used in the clinical settings [72]. Class 2 integrons are included in the $\mathrm{Tn} 7$ family and contain the integrase encoding gene in their structure, followed by gene cassettes. Although it is considered that class 1 integrons have the highest prevalence in A. baumannii isolates, studies have shown a high distribution of class 2. Fonseca et al. highlighted the presence of class 2 integrons in Tn7 in all analyzed isolates. They also observed different gene cassettes in the structure of integrons such as: sat2 (encoding streptothricin resistance), $d f r A l$ (trimethoprim resistance), and aadAl (spectinomycin and streptomycin resistance) [73].

Due to the combined presence of these resistance determinants, few antibiotics remain effective in treating infections caused by this pathogen [62]. For example, in A. baumannii, there have been identified more than 20 types of $\beta$-lactamases, belonging to all four Ambler classes [74-78].

Different studies showed that imipenem-resistant A. baumannii constituted more than $50 \%$ of a worldwide collection of clinical trials between 2005 and 2009 [72]. A surveillance report from Taiwan in 2000 showed that $73 \%$ of isolated A. baumannii collected from 21 medical centers and regional hospitals were resistant to ceftazidime [79]. Lin et al., in 2014, concluded that there are only a few effective anti-Acinetobacter currently available drugs, such as polymyxins and tigecycline [80]. Numerous studies have been performed to understand the pathogenesis and antibiotic resistance mechanisms of A. baumannii strains in response to AR. The difference between Acinetobacter taxonomy using chemotaxonomic methods and phenotypic traits is difficult due to clusters of closely related species. Because antibiotic susceptibility is very different in different genomic species, identification of Acinetobacter spp. is required and very important [62].

\section{Conclusions}

A. baumannii strains can be involved in different types of infections, especially in hospital settings, highlighting the need to implement appropriate infection control measures to limit the spread and decrease the infection rate and mortality. IR mechanisms like natural membrane impermeability, constitutive activity of efflux pumps, and biofilm formation have a significant role in AR of $A$. baumannii. Also, the acquisition and dissemination of ARGs through MGEs add to the acquired resistance in A. baumannii. The enormous adaptability of $A$. baumannii, as well as the ability to survive in extreme environmental conditions, leads to a permanent need to unravel the diversity of mechanisms involved in the acquisition and transfer of resistance determinants. The expansion of the resistant bacteria in clinical settings led to the need for standardization of techniques for the detection of resistant strains. To this end, the transition from laboratory research to entrepreneurial solutions (patenting of new detection techniques) represents a critical approach to counteract the dramatic consequences of bacterial resistance.

\section{Funding}

This research received no external funding. 


\section{Acknowledgments}

This paper was co-financed from the Human Capital Operational Program 2014-2020, project number POCU / 380/6/13/125245 no. 36482 / 23.05.2019 "Excellence in interdisciplinary $\mathrm{Ph} . \mathrm{D}$. and post-Ph.D. research, career alternatives through entrepreneurial initiative (EXCIA)", coordinator The Bucharest University of Economic Studies".

\section{Conflicts of Interest}

The authors declare no conflict of interest.

\section{References}

1. WHO. Antibiotic resistance. https://www.who.int/news-room/fact-sheets/detail/antibiotic-resistance (accessed Jun 2, 2020).

2. Howard, A.; O’Donoghue, M.; Feeney, A.; Sleator, R.D. Acinetobacter Baumannii: An Emerging Opportunistic Pathogen. Virulence 2012, 3, 243-250, https://doi.org/10.4161/viru.19700.

3. Peleg, A.Y.; Seifert, H.; Paterson, D.L. Acinetobacter Baumannii: Emergence of a Successful Pathogen. Clin. Microbiol. Rev. 2008, 21, 538-582, https://doi.org/10.1128/CMR.00058-07.

4. Kulkarni, S.S.; Madalgi, R.; Ajantha, G.S.; Kulkarni, R.D. Identification of Genus Acinetobacter: Standardization of in-House PCR and Its Comparison with Conventional Phenotypic Methods. J. Lab. Physicians 2017, 9, 279-282, https://doi.org/10.4103/0974-2727.214263.

5. Touchon, M.; Cury, J.; Yoon, E.-J.; Krizova, L.; Cerqueira, G.C.; Murphy, C.; Feldgarden, M.; Wortman, J.; Clermont, D.; Lambert, T.; Grillot-Courvalin, C.; Nemec, A.; Courvalin, P.; Rocha, E.P.C. The Genomic Diversification of the Whole Acinetobacter Genus: Origins, Mechanisms, and Consequences. Genome Biol. Evol. 2014, 6, 2866-2882, https://doi.org/10.1093/gbe/evu225.

6. Lessel, E.F. International Committee on Nomenclature of Bacteria Subcommittee on the Taxonomy of Moraxella and Allied Bacteria: Minutes of the Meeting, 11 August 1970. Room Constitution C, Maria-Isabel Hotel, Mexico City, Mexico. Int. J. Syst. Evol. Microbiol. 1971, 21, 213-214, https://doi.org/10.1099/00207713-21-2-213.

7. D’Souza, R.; Pinto, N.A.; Phuong, N. Le; Higgins, P.G.; Vu, T.N.; Byun, J.H.; Cho, Y.L.; Choi, J.R.; Yong, D. Phenotypic and Genotypic Characterization of Acinetobacter Spp. Panel Strains: A Cornerstone to Facilitate Antimicrobial Development. Front. Microbiol. 2019, 10, https://doi.org/10.3389/fmicb.2019.00559.

8. Chen, T.L.; Lee, Y.T.; Kuo, S.C.; Yang, S.P.; Fung, C.P.; Lee, S.D. Rapid Identification of Acinetobacter Baumannii, Acinetobacter Nosocomialis and Acinetobacter Pittii with a Multiplex PCR Assay. J. Med. Microbiol. 2014, 63, 1154-1159, https://doi.org/10.1099/jmm.0.071712-0.

9. Munita, J.M.; Arias, C.A. Mechanisms of Antibiotic Resistance. Microbiol. Spectr. 2016, 4, https://doi.org/10.1128/microbiolspec.VMBF-0016-2015.

10. Rossau, R.; Van Landschoot, A.; Gillis, M.; De Ley, J. Taxonomy of Moraxellaceae Fam. Nov., a New Bacterial Family To Accommodate the Genera Moraxella, Acinetobacter, and Psychrobacter and Related Organisms. Int. J. Syst. Evol. Microbiol. 1991, 41, 310-319, https://doi.org/10.1099/00207713-41-2-310.

11. Erdem, G.; Leber, A. Acinetobacter Species. In: Principles and Practice of Pedriatic Infectious Diseases. Long, S.; Prober, C.; Fischer, M. Eds.; Elsevier, 2017; pp. 851-853.

12. Doughari, H.J.; Ndakidemi, P.A.; Human, I.S.; Benade, S. The Ecology, Biology and Pathogenesis of Acinetobacter Spp.: An Overview. Microbes Environ. 2011, 26, 101-112, https://doi.org/10.1264/jsme2.me10179.

13. Cray, J.A.; Bell, A.N.W.; Bhaganna, P.; Mswaka, A.Y.; Timson, D.J.; Hallsworth, J.E. The Biology of Habitat Dominance; Can Microbes Behave as Weeds? Microb. Biotechnol. 2013, 6, 453-492, https://doi.org/10.1111/1751-7915.12027.

14. Wisplinghoff, H. 181 - Pseudomonas Spp., Acinetobacter Spp. and Miscellaneous Gram-Negative Bacilli. In: Infectious diseases. Cohen, J.; Powderly, W.G.; Opal, S.M.B.T.I.D. Fourth Ed., Eds.; Elsevier, 2017; pp. 1579-1599, https://doi.org/10.1016/B978-0-7020-6285-8.00181-7.

15. Bazzi, W.; Abou Fayad, A.G.; Nasser, A.; Haraoui, L.-P.; Dewachi, O.; Abou-Sitta, G.; Nguyen, V.-K.; Abara, A.; Karah, N.; Landecker, H.; Knapp, C.; McEvoy, M.M.; Zaman, M.H.; Higgins, P.G.; Matar, G.M. Heavy Metal Toxicity in Armed Conflicts Potentiates AMR in A. Baumannii by Selecting for Antibiotic and Heavy Metal Co-Resistance Mechanisms. Front. Microbiol. 2020, 11, https://doi.org/10.3389/fmicb.2020.00068.

16. Al-Kadmy, I.M.S.; Ali, A.N.M.; Salman, I.M.A.; Khazaal, S.S. Molecular Characterization of Acinetobacter Baumannii Isolated from Iraqi Hospital Environment. New microbes new Infect. 2018, 21, 51-57, https://doi.org/10.1016/j.nmni.2017.10.010. 
17. McConnell, M.J.; Actis, L.; Pachón, J. Acinetobacter Baumannii: Human Infections, Factors Contributing to Pathogenesis and Animal Models. FEMS Microbiol. Rev. 2013, 37, 130-155, https://doi.org/10.1111/j.1574-6976.2012.00344.x.

18. Gallego, L. Acinetobacter Baumannii: Factors Involved in Its High Adaptability to Adverse Environmental Conditions. Microb. Exp. 2016, 3, https://doi.org/10.15406/jmen.2016.03.00085.

19. Joly-Guillou, M.L. Clinical Impact and Pathogenicity of Acinetobacter. Clin. Microbiol. Infect. Off. Publ. Eur. Soc. Clin. Microbiol. Infect. Dis. 2005, 11, 868-873, https://doi.org/10.1111/j.14690691.2005.01227.x.

20. Dijkshoorn, L.; Nemec, A.; Seifert, H. An Increasing Threat in Hospitals: Multidrug-Resistant Acinetobacter Baumannii. Nat. Rev. Microbiol. 2007, 5, 939-951, https://doi.org/10.1038/nrmicro1789.

21. Asif, M.; Alvi, I.A.; Rehman, S.U. Insight into Acinetobacter Baumannii: Pathogenesis, Global Resistance, Mechanisms of Resistance, Treatment Options, and Alternative Modalities. Infect. Drug Resist. 2018, 11, 1249-1260, https://doi.org/10.2147/IDR.S166750.

22. Wong, D.; Nielsen, T.B.; Bonomo, R.A.; Pantapalangkoor, P.; Luna, B.; Spellberg, B. Clinical and Pathophysiological Overview of Acinetobacter Infections: A Century of Challenges. Clin. Microbiol. Rev. 2017, 30, 409-447, https://doi.org/10.1128/CMR.00058-16.

23. Xue, L.Y.; Gaowa, S.; Wang, W.; Zhao, F.; Zhu, H.C.; Yu, X.Y.; Gong, Y. Ventilator-Associated Pneumonia in Patients with Cerebral Hemorrhage: Impact on Mortality and Microbiological Characterization. Med. Clin. (Barc). 2020, 154, 400-405, https://doi.org/10.1016/j.medcli.2020.01.003.

24. Vaneechoutte, M.; Devriese, L.A.; Dijkshoorn, L.; Lamote, B.; Deprez, P.; Verschraegen, G.; Haesebrouck, F. Acinetobacter Baumannii-Infected Vascular Catheters Collected from Horses in an Equine Clinic. J. Clin. Microbiol. 2000, 38, 4280-4281.

25. Francey, T.; Gaschen, F.; Nicolet, J.; Burnens, A.P. The Role of Acinetobacter Baumannii as a Nosocomial Pathogen for Dogs and Cats in an Intensive Care Unit. J. Vet. Intern. Med. 2000, 14, 177-183.

26. Endimiani, A.; Hujer, K.M.; Hujer, A.M.; Bertschy, I.; Rossano, A.; Koch, C.; Gerber, V.; Francey, T.; Bonomo, R.A.; Perreten, V. Acinetobacter Baumannii Isolates from Pets and Horses in Switzerland: Molecular Characterization and Clinical Data. J. Antimicrob. Chemother. 2011, 66, 2248-2254, https://doi.org/10.1093/jac/dkr289.

27. Ramirez, M.S.; Bonomo, R.A.; Tolmasky, M.E. Carbapenemases: Transforming Acinetobacter Baumannii into a Yet More Dangerous Menace. Biomolecules 2020, 10. https://doi.org/10.3390/biom10050720.

28. Vázquez-López, R.; Solano-Gálvez, S.G.; Juárez Vignon-Whaley, J.J.; Abello Vaamonde, J.A.; Padró Alonzo, L.A.; Rivera Reséndiz, A.; Muleiro Álvarez, M.; Vega López, E.N.; Franyuti-Kelly, G.; ÁlvarezHernández, D.A.; Moncaleano Guzmán, V.; Juárez Bañuelos, J.E.; Marcos Felix, J.; González Barrios, J.A.; Barrientos Fortes, T. Acinetobacter Baumannii Resistance: A Real Challenge for Clinicians. Antibiot. (Basel, Switzerland) 2020, 9, https://doi.org/10.3390/antibiotics9040205.

29. Santajit, S.; Indrawattana, N. Mechanisms of Antimicrobial Resistance in ESKAPE Pathogens. Biomed Res. Int. 2016, 2016, https://doi.org/10.1155/2016/2475067.

30. Ruppé, É.; Woerther, P.L.; Barbier, F. Mechanisms of Antimicrobial Resistance in Gram-Negative Bacilli. Ann. Intensive Care 2015, 5, https://doi.org/10.1186/s13613-015-0061-0.

31. Ogbolu, D.O.; Alli, O.A.T.; Oluremi, A.S.; Ogunjimi, Y.T.; Ojebode, D.I.; Dada, V.; Alaka, O.O.; FosterNyarko, E.; Webber, M.A. Contribution of NDM and OXA-Type Carbapenemases to Carbapenem Resistance in Clinical Acinetobacter Baumannii from Nigeria. Infect. Dis. (London, England) 2020, 1-7, https://doi.org/10.1080/23744235.2020.1775881.

32. Mentasti, M.; Prime, K.; Sands, K.; Khan, S.; Wootton, M. Rapid Detection of OXA-23-like, OXA-24-like and OXA-58-like Carbapenemases from Acinetobacter Species by Real-Time PCR. J. Hosp. Infect. 2020, https://doi.org/10.1016/j.jhin.2020.06.015.

33. Bonomo, R.A.; Szabo, D. Mechanisms of Multidrug Resistance in Acinetobacter Species and Pseudomonas Aeruginosa. Clin. Infect. Dis. 2006, 43 Suppl 2, S49-56, https://doi.org/10.1086/504477.

34. Damier-Piolle, L.; Magnet, S.; Brémont, S.; Lambert, T.; Courvalin, P. AdeIJK, a Resistance-NodulationCell Division Pump Effluxing Multiple Antibiotics in Acinetobacter Baumannii. Antimicrob. Agents Chemother. 2008, 52, 557-562, https://doi.org/10.1128/AAC.00732-07.

35. Smani, Y.; Fàbrega, A.; Roca, I.; Sánchez-Encinales, V.; Vila, J.; Pachón, J. Role of OmpA in the Multidrug Resistance Phenotype of Acinetobacter Baumannii. Antimicrob. Agents Chemother. 2014, 58, 1806-1808, https://doi.org/10.1128/AAC.02101-13.

36. Sánchez-Encinales, V.; Álvarez-Marín, R.; Pachón-Ibáñez, M.E.; Fernández-Cuenca, F.; Pascual, A.; Garnacho-Montero, J.; Martínez-Martínez, L.; Vila, J.; Tomás, M.M.; Cisneros, J.M.; Bou, G.; RodríguezBaño, J.; Pachón, J.; Smani, Y. Overproduction of Outer Membrane Protein A by Acinetobacter Baumannii as a Risk Factor for Nosocomial Pneumonia, Bacteremia, and Mortality Rate Increase. J. Infect. Dis. 2017, 215, 966-974, https://doi.org/10.1093/infdis/jix010.

37. Sato, Y.; Unno, Y.; Kawakami, S.; Ubagai, T.; Ono, Y. Virulence Characteristics of Acinetobacter Baumannii Clinical Isolates Vary with the Expression Levels of Omps. J. Med. Microbiol. 2017, 66, 203212, https://doi.org/10.1099/jmm.0.000394. 
38. Gunn, J.S.; Bakaletz, L.O.; Wozniak, D.J. What's on the Outside Matters: The Role of the Extracellular Polymeric Substance of Gram-Negative Biofilms in Evading Host Immunity and as a Target for Therapeutic Intervention. J. Biol. Chem. 2016, 291, 12538-12546, https://doi.org/10.1074/jbc.R115.707547.

39. Sun, F.; Qu, F.; Ling, Y.; Mao, P.; Xia, P.; Chen, H.; Zhou, D. Biofilm-Associated Infections: Antibiotic Resistance and Novel Therapeutic Strategies. Future Microbiol. 2013, 8, 877-886, https://doi.org/10.2217/fmb.13.58.

40. Yang, J.; Toyofuku, M.; Sakai, R.; Nomura, N. Influence of the Alginate Production on Cell-to-Cell Communication in Pseudomonas Aeruginosa PAO1. Environ. Microbiol. Rep. 2017, 9, 239-249, https://doi.org/10.1111/1758-2229.12521.

41. Moreno-Gámez, S.; Sorg, R.A.; Domenech, A.; Kjos, M.; Weissing, F.J.; van Doorn, G.S.; Veening, J.W. Quorum Sensing Integrates Environmental Cues, Cell Density and Cell History to Control Bacterial Competence. Nat. Commun. 2017, 8, https://doi.org/10.1038/s41467-017-00903-y.

42. Stewart, P.S.; Costerton, J.W. Antibiotic Resistance of Bacteria in Biofilms. Lancet (London, England) 2001, 358, 135-138, https://doi.org/10.1016/s0140-6736(01)05321-1.

43. Gilbert, P.; McBain, A.J. Potential Impact of Increased Use of Biocides in Consumer Products on Prevalence of Antibiotic Resistance. Clin. Microbiol. Rev. 2003, 16, 189-208, https://doi.org/10.1128/cmr.16.2.189208.2003.

44. Lin, M.F.; Lin, Y.Y.; Lan, C.Y. Characterization of Biofilm Production in Different Strains of Acinetobacter Baumannii and the Effects of Chemical Compounds on Biofilm Formation. PeerJ 2020, 8, https://doi.org/10.7717/peerj.9020.

45. Kaviani, R.; Pouladi, I.; Niakan, M.; Mirnejad, R. Molecular Detection of Adefg Efflux Pump Genes and Their Contribution to Antibiotic Resistance in Acinetobacter Baumannii Clinical Isolates. Reports Biochem. Mol. Biol. 2020, 8, 413-418.

46. Pagedar, A.; Singh, J.; Batish, V.K. Adaptation to Benzalkonium Chloride and Ciprofloxacin Affects Biofilm Formation Potential, Efflux Pump and Haemolysin Activity of Escherichia Coli of Dairy Origin. $J$. Dairy Res. 2012, 79, 383-389, https://doi.org/10.1017/S0022029912000295.

47. Siguier, P.; Gourbeyre, E.; Chandler, M. Bacterial Insertion Sequences: Their Genomic Impact and Diversity. FEMS Microbiol. Rev. 2014, 38, 865-891, https://doi.org/10.1111/1574-6976.12067.

48. Bennett, P.M. Plasmid Encoded Antibiotic Resistance: Acquisition and Transfer of Antibiotic Resistance Genes in Bacteria. Br. J. Pharmacol. 2008, 153 Suppl (Suppl 1), S347-57, https://doi.org/10.1038/sj.bjp.0707607.

49. Partridge, S.R.; Kwong, S.M.; Firth, N.; Jensen, S.O. Mobile Genetic Elements Associated with Antimicrobial Resistance. Clin. Microbiol. Rev. 2018, 31, https://doi.org/10.1128/CMR.00088-17.

50. Ilangovan, A.; Connery, S.; Waksman, G. Structural Biology of the Gram-Negative Bacterial Conjugation Systems. Trends Microbiol. 2015, 23, 301-310, https://doi.org/10.1016/j.tim.2015.02.012.

51. Frost, L.S.; Leplae, R.; Summers, A.O.; Toussaint, A. Mobile Genetic Elements: The Agents of Open Source Evolution. Nat. Rev. Microbiol. 2005, 3, 722-732, https://doi.org/10.1038/nrmicro1235.

52. Carattoli, A. Plasmids and the Spread of Resistance. Int. J. Med. Microbiol. 2013, 303, 298-304, https://doi.org/10.1016/j.ijmm.2013.02.001.

53. Shintani, M.; Sanchez, Z.K.; Kimbara, K. Genomics of Microbial Plasmids: Classification and Identification Based on Replication and Transfer Systems and Host Taxonomy. Front. Microbiol. 2015, 6, https://doi.org/10.3389/fmicb.2015.00242.

54. Bertini, A.; Poirel, L.; Mugnier, P.D.; Villa, L.; Nordmann, P.; Carattoli, A. Characterization and PCR-Based Replicon Typing of Resistance Plasmids in Acinetobacter Baumannii. Antimicrob. Agents Chemother. 2010, 54, 4168-4177, https://doi.org/10.1128/AAC.00542-10.

55. Pagano, M.; Martins, A.F.; Barth, A.L. Mobile Genetic Elements Related to Carbapenem Resistance in Acinetobacter Baumannii. Brazilian J. Microbiol. [publication Brazilian Soc. Microbiol. 2016, 47, 785792, https://doi.org/10.1016/j.bjm.2016.06.005.

56. Singh, M.; De Silva, P.M.; Al-Saadi, Y.; Switala, J.; Loewen, P.C.; Hausner, G.; Chen, W.; Hernandez, I.; Castillo-Ramirez, S.; Kumar, A. Characterization of Extremely Drug-Resistant and Hypervirulent Acinetobacter Baumannii AB030. Antibiot. (Basel, Switzerland) 2020, 9, https://doi.org/10.3390/antibiotics9060328.

57. Villalón, P.; Valdezate, S.; Medina-Pascual, M.J.; Carrasco, G.; Vindel, A.; Saez-Nieto, J.A. Epidemiology of the Acinetobacter-Derived Cephalosporinase, Carbapenem-Hydrolysing Oxacillinase and Metallo- $\beta$ Lactamase Genes, and of Common Insertion Sequences, in Epidemic Clones of Acinetobacter Baumannii from Spain. J. Antimicrob. Chemother. 2013, 68, 550-553, https://doi.org/10.1093/jac/dks448.

58. Poirel, L.; Bonnin, R.A.; Nordmann, P. Analysis of the Resistome of a Multidrug-Resistant NDM-1Producing Escherichia Coli Strain by High-Throughput Genome Sequencing. Antimicrob. Agents Chemother. 2011, 55, 4224-4229, https://doi.org/10.1128/AAC.00165-11.

59. Segal, H.; Garny, S.; Elisha, B.G. Is IS(ABA-1) Customized for Acinetobacter? FEMS Microbiol. Lett. 2005, 243, 425-429, https://doi.org/10.1016/j.femsle.2005.01.005.

60. de Freitas, S.B.; Amaral, S.C.; Ferreira, M.R.A.; Roloff, B.C.; Moreira, C.J.; Conceição, F.R.; Hartwig, D.D. Molecular Characterization of Carbapenem-Resistant Acinetobacter Baumannii Associated with 
Nosocomial Infection in the Pelotas, RS, Brazil. Curr. Microbiol. 2020, https://doi.org/10.1007/s00284-02002060-w.

61. Turton, J.F.; Ward, M.E.; Woodford, N.; Kaufmann, M.E.; Pike, R.; Livermore, D.M.; Pitt, T.L. The Role of ISAba1 in Expression of OXA Carbapenemase Genes in Acinetobacter Baumannii. FEMS Microbiol. Lett. 2006, 258, 72-77, https://doi.org/10.1111/j.1574-6968.2006.00195.x.

62. Lee, Y.; Kim, C.K.; Lee, H.; Jeong, S.H.; Yong, D.; Lee, K. A Novel Insertion Sequence, ISAba10, Inserted into ISAbal Adjacent to the Bla(OXA-23) Gene and Disrupting the Outer Membrane Protein Gene CarO in Acinetobacter Baumannii. Antimicrob. Agents Chemother. 2011, 55, 361-363, https://doi.org/10.1128/AAC.01672-09.

63. Toleman, M.A.; Walsh, T.R. Combinatorial Events of Insertion Sequences and ICE in Gram-Negative Bacteria. FEMS Microbiol. Rev. 2011, 35, 912-935, https://doi.org/10.1111/j.1574-6976.2011.00294.x.

64. van der Zee, A.; Kraak, W.B.; Burggraaf, A.; Goessens, W.H.F.; Pirovano, W.; Ossewaarde, J.M.; Tommassen, J. Spread of Carbapenem Resistance by Transposition and Conjugation Among Pseudomonas Aeruginosa. Front. Microbiol. 2018, 9, https://doi.org/10.3389/fmicb.2018.02057.

65. Mugnier, P.D.; Poirel, L.; Naas, T.; Nordmann, P. Worldwide Dissemination of the BlaOXA-23 Carbapenemase Gene of Acinetobacter Baumannii. Emerg. Infect. Dis. 2010, 16, 35-40, https://doi.org/10.3201/eid1601.090852.

66. Rowe-Magnus, D.A.; Mazel, D. Integrons: Natural Tools for Bacterial Genome Evolution. Curr. Opin. Microbiol. 2001, 4, 565-569, https://doi.org/10.1016/s1369-5274(00)00252-6.

67. Leungtongkam, U.; Thummeepak, R.; Tasanapak, K.; Sitthisak, S. Acquisition and Transfer of Antibiotic Resistance Genes in Association with Conjugative Plasmid or Class 1 Integrons of Acinetobacter Baumannii. PLoS One 2018, 13, https://doi.org/10.1371/journal.pone.0208468.

68. Cameranesi, M.M.; Morán-Barrio, J.; Limansky, A.S.; Repizo, G.D.; Viale, A.M. Site-Specific Recombination at XerC/D Sites Mediates the Formation and Resolution of Plasmid Co-Integrates Carrying a Bla(OXA-58)- and TnaphA6-Resistance Module in Acinetobacter Baumannii. Front. Microbiol. 2018, 9 , https://doi.org/10.3389/fmicb.2018.00066.

69. Gillings, M.R. Integrons: Past, Present, and Future. Microbiol. Mol. Biol. Rev. 2014, 78, 257-277, https://doi.org/10.1128/MMBR.00056-13.

70. Lee, Y.T.; Huang, L.Y.; Chen, T.L.; Siu, L.K.; Fung, C.P.; Cho, W.L.; Yu, K.W.; Liu, C.Y. Gene Cassette Arrays, Antibiotic Susceptibilities, and Clinical Characteristics of Acinetobacter Baumannii Bacteremic Strains Harboring Class 1 Integrons. J. Microbiol. Immunol. Infect. 2009, 42, 210-219.

71. Fluit, A.C.; Schmitz, F.J. Resistance Integrons and Super-Integrons. Clin. Microbiol. Infect. Off. Publ. Eur. Soc. Clin. Microbiol. Infect. Dis. 2004, 10, 272-288, https://doi.org/10.1111/j.1198-743X.2004.00858.x.

72. Mendes, R.E.; Castanheira, M.; Toleman, M.A.; Sader, H.S.; Jones, R.N.; Walsh, T.R. Characterization of an Integron Carrying BlaIMP-1 and a New Aminoglycoside Resistance Gene, Aac(6')-31, and Its Dissemination among Genetically Unrelated Clinical Isolates in a Brazilian Hospital. Antimicrob. Agents Chemother. 2007, 51, 2611-2614, https://doi.org/10.1128/AAC.00838-06.

73. Fonseca, É.L.; Freitas, F. dos S.; Scheidegger, É.M.D.; Jacinto, T.; Vicente, A.C.P. Class 2 Integrons in Multidrug-Resistant Acinetobacter Baumannii Circulating in Different Brazilian Geographic Regions. International journal of antimicrobial agents. 2011, 38, 95-96, https://doi.org/10.1016/j.ijantimicag.2011.03.013.

74. Vrancianu, C.O.; Popa, L.I.; Bleotu, C.; Chifiriuc, M.C. Targeting Plasmids to Limit Acquisition and Transmission of Antimicrobial Resistance. Front. Microbiol. 2020, 11, https://doi.org/10.3389/fmicb.2020.00761.

75. Vrancianu, C.O.; Gheorghe, I.; Czobor, I.B.; Chifiriuc, M.C. Antibiotic Resistance Profiles, Molecular Mechanisms and Innovative Treatment Strategies of Acinetobacter Baumannii. Microorganisms 2020, 8, https://doi.org/10.3390/microorganisms8060935.

76. Gheorghe I., Cristea V.C., Marutescu L., Popa M., Murariu C., Trusca B.S., Borcan E., Ghita M.C., Lazar V., Chifiriuc M.C. Resistance and Virulence Features in Carbapenem-Resistant Acinetobacter Baumannii Community Acquired and Nosocomial Isolates in Romania. Rev. Chim. 2019, 70, 3502-3507, https://doi.org/10.37358/RC.19.10.7584.

77. Gheorghe, I.; Novais, Â.; Grosso, F.; Rodrigues, C.; Chifiriuc, M.C.; Lazar, V.; Peixe, L. Snapshot on Carbapenemase-Producing Pseudomonas Aeruginosa and Acinetobacter Baumannii in Bucharest Hospitals Reveals Unusual Clones and Novel Genetic Surroundings for BlaOXA-23. J. Antimicrob. Chemother. 2015, 70, 1016-1020, https://doi.org/10.1093/jac/dku527.

78. Mihai, D.; Chifiruc, M.C.; Gheorghe, I.; Ditu, L.; Mihaescu, G. Resistance and Virulence Gene Profiles in Klebsiella pneumoniae and Acinetobacter baumannii Strains Isolated from Hospitalized Patients. International Multidisciplinary Scientific GeoConference: $\quad$ SGEM; $\quad$ Sofia 2018, https://doi.org/10.5593/sgem2018V/6.4/S08.034.

79. Lauderdale, T.L.; Clifford McDonald, L.; Shiau, Y.R.; Chen, P.C.; Wang, H.Y.; Lai, J.F.; Ho, M. The Status of Antimicrobial Resistance in Taiwan among Gram-Negative Pathogens: The Taiwan Surveillance of Antimicrobial Resistance (TSAR) Program, 2000. Diagn. Microbiol. Infect. Dis. 2004, 48, 211-219, https://doi.org/10.1016/j.diagmicrobio.2003.10.005. 
80. Lin, M.F.; Lin, Y.Y.; Yeh, H.W.; Lan, C.Y. Role of the BaeSR Two-Component System in the Regulation of Acinetobacter Baumannii AdeAB Genes and Its Correlation with Tigecycline Susceptibility. BMC Microbiol. 2014, 14, https://doi.org/10.1186/1471-2180-14-119. 